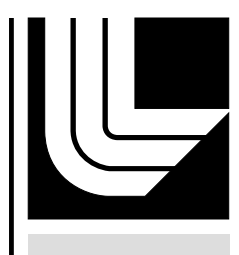

LAW RENCE LIVERMORE N A T IO N A L LABORATORY

\title{
UCRL-TR-217843
}

\section{Particle Interactions in DNA-laden Flows}

M. D. Bybee, G. H. Miller, D. Trebotich

December 21, 2005 
This document was prepared as an account of work sponsored by an agency of the United States Government. Neither the United States Government nor the University of California nor any of their employees, makes any warranty, express or implied, or assumes any legal liability or responsibility for the accuracy, completeness, or usefulness of any information, apparatus, product, or process disclosed, or represents that its use would not infringe privately owned rights. Reference herein to any specific commercial product, process, or service by trade name, trademark, manufacturer, or otherwise, does not necessarily constitute or imply its endorsement, recommendation, or favoring by the United States Government or the University of California. The views and opinions of authors expressed herein do not necessarily state or reflect those of the United States Government or the University of California, and shall not be used for advertising or product endorsement purposes.

This work was performed under the auspices of the U.S. Department of Energy by University of California, Lawrence Livermore National Laboratory under Contract W-7405-Eng-48. 


\title{
Particle Interactions in DNA-laden Flows
}

\author{
Michael Bybee \\ Greg Miller \\ David Trebotich
}

December 20, 2005

\section{Introduction}

Microfluidic devices are becoming state-of-the-art in many significant applications including pathogen detection, continuous monitoring, and drug delivery. Numerical algorithms which can simulate flows of complex fluids within these devices are needed for their development and optimization. A method is being developed at LLNL by Trebotich et. al. [30] for simulations of DNA-laden flows in complex microscale geometries such as packed bed reactors and pillar chips.

In this method an incompressible Newtonian fluid is discretized with Cartesian grid embedded boundary methods, and the DNA is represented by a bead-rod polymer model. The fluid and polymer are coupled through a body force.

In its current state, polymer-surface interactions are treated as elastic collisions between beads and surface, and polymer-polymer interactions are neglected. Implementation of polymer-polymer interactions is the main objective of this work. It is achieved by two methods: 1) a rigid constraint whereby rods elastically bounce off one another, and 2) a smooth potential acting between rods. In addition, a smooth potential is also implemented for the polymer-surface interactions.

Background information will also be presented as well as related work by other researchers. 


\section{Physical Characteristics of DNA}

Double stranded DNA has a width of $2 \mathrm{~nm}$ and contour length of $0.34 \mathrm{~nm}$ per base pair (refers to the $\mathrm{B}$ form which is most abundant in nature). The average mass of a DNA base pair is about $10^{-21}$ grams.

\section{Coarse Grained Polymer Models}

Polymers can be modeled from first principles via ab initio simulations. Coarse grained models are necessary for the simulation of longer polymers for longer times. The most basic coarse grained model would be molecular dynamics in which atomic interactions are approximated by empirical force fields (i.e. Lennard Jones). A slightly more coarse grained version of molecular dynamics is achieved by using force fields to approximate the interactions between groups of atoms. Even more coarse grained models include the bead-rod model, bead-spring model, and worm like chain. For a good review on numerical algorithms and coarse-graining issues involved with Brownian dynamics simulations of bead-rod and bead-spring chains, see Somasi et. al. [28].

\subsection{Bead-Rod Model}

"In many aspects, sufficiently long polymers behave similar to an idealized chain of $n$ segments of length $l_{k}$, where the chain segments are not restricted in their torsional movement with respect to one another. Such a chain is termed a Gaussian or freely jointed chain (FJC). The parameter $l_{k}$ is called the statistical segment length or Kuhn length after the Swiss scientist Werner Kuhn who developed the concept and much of the theoretical description of the FJC model in the 1930s" [25]. A related parameter is the persistence length which is equal to half the Kuhn length.

The Kuhn length and persistence length are measures of the stiffness of a polymer. For example, polyethylene glycol is a very flexible polymer with a Kuhn length of $0.76 \mathrm{~nm}$ [13]. Single stranded DNA is stiffer with a Kuhn length of 2-6 nm, and double stranded DNA is extremely stiff with a Kuhn length of $100 \mathrm{~nm}$ [25]. It should also be noted that these characteristic lengths are temperature dependent and can be dependent on salt concentration in the case of charged polymers. 
The bead-rod model was proposed by Kramers [15]. The polymer is modeled by a series of beads connected by rigid rods. The beads are treated as point masses which experience viscous drag and thermal bombardment by solvent molecules. The rods simply serve to maintain a constant distance (the Kuhn length) between adjacent beads and experience no effects from the solvent. Many Brownian dynamics simulations for bead-rod models follow the formulations outlined by Liu [19] and Doyle [6]. Constant rod lengths can be maintained using a Lagrange multiplier technique outlined by Ciccotti, et. al. [3] [4] [32]. Somasi, et. al. [28] found that in solving the nonlinear system resulting from the constraints, the Picard iteration proposed by Liu is more efficient than Newton's Method.

\subsection{Bead-Spring Model}

In the bead-spring model, several rods are replaced by an entropic spring. The number of rods that can be equivalently replaced by an entropic spring depends on the flow strength [28].

\section{Polymer-Polymer and Polymer-Surface In- teractions}

Polymer-polymer and polymer-surface interactions result from excluded volume, electrostatic, and other forces such as dispersion forces. An important aspect of such interactions is that they prevent unphysical crossings.

\subsection{Excluded-Volume Forces}

Excluded-volume forces are short-ranged repulsive forces due to overlapping electron clouds. They have been modeled extensively in Brownian dynamics simulations with bead-bead repulsions and spring-spring (or rod-rod) repulsions [16]. These repulsions often use a purely repulsive Lennard-Jones potential although some have suggested using a softer repulsive potential [21]. It should be noted that excluded-volume forces result in a swelling of the polymer.

Spring-spring repulsions have two advantages over bead-bead repulsions. First, spring-spring repulsions guarantee that springs will not cross while bead-bead repulsions simply make spring crossings energetically unfavorable. 
Second, spring-spring repulsions do not place a limit on the size of springs while bead-bead repulsions require small springs because the range of the repulsive force is necessarily of the order of the maximum spring length.

\subsection{Electrostatic Forces}

Two point charges in a vacuum will interact via a long-ranged Coulomb potential given by

$$
U^{\mathrm{C}}=\frac{q_{1} q_{2}}{4 \pi \epsilon_{0}} \frac{1}{r},
$$

where $q_{1}$ and $q_{2}$ are the charges, $r$ is the distance between them, and $\epsilon_{0}$ is the permittivity of free space.

If the point charges are placed in a dielectric medium, the interaction is screened due to the polarization of the particles of the dielectric medium. It still maintains its long-ranged character, but is reduced by a dimensionless factor called the dielectric constant $\epsilon$ so that the screened Coulomb potential is given by

$$
U^{\mathrm{C}}=\frac{q_{1} q_{2}}{4 \pi \epsilon \epsilon_{0}} \frac{1}{r}
$$

Further screening of the Coulomb interaction will occur if the medium contains ions (even pure water contains ions). The mathematics become very complicated, but essentially, the Coulomb interaction decays exponentially and becomes negligible beyond a separation characterized by the Debye screening length which is given by

$$
\kappa^{-1}=\left(\frac{\epsilon \epsilon_{0} k T}{\sum q_{i}^{2} n_{i}}\right)^{1 / 2},
$$

where $k$ is Boltzmann's constant, $T$ is the absolute temperature, and $q_{i}$ and $n_{i}$ are the charge and concentration of the $i^{\text {th }}$ ionic species respectively. (see Russel [26]). The Debye-Hückel theory is commonly used and is of the form

$$
U^{\mathrm{DH}}=A \frac{e^{-\kappa r}}{r} .
$$

Along the backbone of DNA there are two negatively charged phosphate groups per base pair. Positive ions in solution are attracted to these negative charges and form an electric double layer whose thickness is given by the Debye screening length. In physiological conditions $\left(\sim 10^{-1} \mathrm{M}\right)$, the Debye 
length is on the order of $1 \mathrm{~nm}$. In pure water $\left(\sim 10^{-7} \mathrm{M}\right)$, the Debye screening length is on the order of $1 \mu \mathrm{m}$.

Because DNA is negatively charged, it will move in the presence of an electric field. For more information on the electrophoresis of DNA, see Viovy $[31]$.

\subsection{Other Forces}

Dispersion forces refer to short-ranged attractive forces which occur between two dipoles (Keesom), a dipole and an induced-dipole (Debye), and two induced-dipoles (van der Waals or London). There are many other forces including hydrogen-bonding, hydrophobic, hydrophilic, and solvation forces. These forces become important at separations less than a couple of nanometers [17].

\subsection{Summary}

For simulations of DNA in physiological conditions, all of the above forces involved in polymer-polymer and polymer-surface interactions act over length scales significantly smaller than a rod length (Kuhn step). Furthermore, the dynamics of long DNA in microdevices might be dominated by the drag forces exerted by the fluid. Therefore, the details of the polymer-polymer and polymer-surface interactions might not be very important so long as they prevent unphysical crossings.

Padding and Briels [23] describe a complex algorithm to detect and prevent bond crossings. Bonds are considered as elastic bands between bonded particles. When any two of these elastic bands make contact, an entanglement point is created which prevents them from crossing.

This work has developed two methods for preventing rod-rod crossings. The first is a rigid constraint whereby infinitely thin rods elastically bounce off one another. The second is a short-ranged Debye-Hückel potential acting between rods. The short-ranged Debye-Hückel potential has also been implemented for rod-surface interactions. These algorithms are explained in detail in Section 6. 


\section{Related Work}

This section presents some work by other researchers that is related to this work. It is by no means comprehensive.

\subsection{General}

Schlick gives a review on the computational challenges of simulating large DNA over long times [27]

\subsection{Simulation of DNA in Microfluidic Devices}

Martin Streek's Ph.D. thesis is titled "Brownian Dynamics Simulation of Migration of DNA in Structured Microchannels" [29].

Patel and Shaqfeh performed Brownian dynamics simulations of DNA separation by post arrays [24]. They employed a bead-rod model with a Lennard-Jones repulsive potential for bead-post interactions. Post diameter equal to Kuhn length. Adaptive time stepping. Makes references to others who have also done these types of simulations and experiments.

Jendrejack et. al. studied the effects of confinement on DNA dynamics in microfluidic devices [11] [9] [10] [7] [8]. They couple a Brownian dynamics simulation to a finite element method for incompressible Stokes flow. The DNA is represented by a bead-spring polymer model with an excluded volume potential between beads and a bead-wall repulsive potential. Bead-bead hydrodynamic interactions are incorporated through the RotnePrager-Yamakawa (RPY) tensor while bead-wall hydrodynamic interactions are incorporated through the finite element solution. The RPY tensor is the most common method of including hydrodynamic interactions although it only accounts for two-body effects. Jendrejack's 2003 Ph.D. thesis is titled "Multiscale Simulations of Dilute-Solution Macromolecular Dynamics in Macroscopic and Microscopic Geometries."

Woo, Shaqfeh, and Khomami discuss the effects of confinement on dynamics and rheology of dilute DNA solutions in [34] and [33].

\subsection{Hydrodynamic Interactions and Polymers}

Butler and Shaqfeh performed the first Brownian dynamics simulations of polymers with multibody hydrodynamic interactions [2]. The polymer is 
composed of rigid rods modeled with the slender-body theory of Batchelor. Hydrodynamic resistance is distributed along the entire contour length of the polymer in a continuous manner rather than concentrating the resistance at localized positions. Incorporates rod-rod repulsions. Compares results from different levels of approximation of the hydrodynamic interactions including two-body, multi-body, and the freely draining case. Does not explicitly state whether lubrication interactions are included.

\subsection{Coupled Particle-Fluid Methods}

Methods for simulating coupled particle-fluid flows include finite-element with moving boundaries, distributed Lagrange multiplier, immersed boundary, Lattice-Boltzmann, boundary integral, multipole (Stokesian Dynamics and particle-mesh Ewald), and force-coupling [5].

The work by Jendrejack mentioned in Section 5.2.

In the force-coupling method, the Navier-Stokes equations are solved subject to a body force resulting from finite force multipoles. Unlike the method of Jendrejack above, it also attempts to account for the no-slip condition of the particle itself. Its application to particulate microflows in Stokes flow and low Reynolds number flow is discussed in [18]. Incorporation of lubrication effects is discussed in [5]. Experimental verification is discussed in [20].

\subsection{Experimental Validation}

Jian and Vologodskii claim to have developed a "carefully parameterized and tested simulation procedure for studying the dynamic properties of long linear DNA" verified with experimental data. Performed simulations up to 2311 base pairs [12].

\subsection{Other}

Klenin, Merlitz, and Langowski present second-order explicit Brownian dynamics algorithm including stretching, bending, and twisting potentials as well as accounting for electrostatics and hydrodynamic interactions. The code is available upon request [14].

Mielke, et.al. also present a Brownian dynamics algorithm with stretching, bending, and twisting potentials and excluded volume [22]. 


\section{Algorithms}

The two methods for preventing rod-rod crossings are presented here. While not implemented in this work, there are efficient algorithms which alleviate the inherent $O\left(n^{2}\right)$ scaling of these methods. For short-ranged interactions, a Verlet neighbor list can be employed [1]. For long-ranged interactions, several Ewald techniques are available.

In addition, an error in the algorithm presented in [30] is discussed.

\subsection{Rod-rod Uncrossability Constraint}

The rod-rod uncrossability constraint works by detecting rod-rod collisions and treating them as elastic collisions between infinitely thin rods. It is similar to the bead-surface uncrossability constraint already implemented in [30]. The algorithm is outlined below as an extension to the existing algorithm in [30]. Steps 1, 2, and 4 are described in more detail in [30]. The ideas for steps 3.1-3.4 have been taken from [23].

The polymer is represented by a series of beads connected by rigid rods. The bead positions and velocities are given by $\boldsymbol{x}$ and $\boldsymbol{v}$ respectively. Rod $i$ is defined as the line segment from bead $i$ to bead $i+1$, or from $\boldsymbol{x}_{i}$ to $\boldsymbol{x}_{i+1}$.

For each time step, beginning with $\boldsymbol{x}^{n}$ and $\boldsymbol{v}^{n}$ :

1. Calculate the unconstrained motion to obtain $\boldsymbol{x}^{*}$ and $\boldsymbol{v}^{*}$.

2. Calculate the motion subject to the rod length constraint to obtain $\boldsymbol{x}^{\dagger}$ and $\boldsymbol{v}^{\dagger}$.

3. Calculate the motion subject to the rod-rod uncrossability constraint to obtain $\boldsymbol{x}^{\ddagger}$ and $\boldsymbol{v}^{\ddagger}$. The details of this step are presented here.

Calculate $\boldsymbol{v}^{\Delta t}$, the bead velocities over the current time step

$$
\boldsymbol{v}^{\Delta t}=\left(\boldsymbol{x}^{\dagger}-\boldsymbol{x}^{n}\right) / \Delta t
$$

so that the time-linear trajectory of each bead over the current time step is

$$
\boldsymbol{x}=\boldsymbol{x}^{n}+\boldsymbol{v}^{\Delta t} t \quad t \in(0, \Delta t)
$$

Repeatedly loop through all rod pairs until no more collisions are detected. For each pair of rods $i$ and $j$ : 
3a. Calculate the triple product $V_{i j}$ at times 0 and $\Delta t$ where

$$
V_{i j}=\left(\boldsymbol{x}_{i}-\boldsymbol{x}_{j}\right) \cdot\left(\left(\boldsymbol{x}_{i+1}-\boldsymbol{x}_{i}\right) \times\left(\boldsymbol{x}_{j+1}-\boldsymbol{x}_{j}\right)\right)
$$

The value of $V_{i j}$ will be zero if the infinite lines containing the rods intersect or are parallel. Therefore, if the value of $V_{i j}$ changes sign over the time step, a possible rod-rod crossing has occurred. Otherwise, proceed to step $3 \mathrm{k}$.

3b. Calculate $\tau$, the time of crossing. Substituting the time-linear trajectories of (6) into (7) gives a third-order polynomial in $t$ for $V_{i j}$. The smallest root of this polynomial in the range $(0, \Delta t)$ will be $\tau$.

To simplify the calculation, let

$$
\begin{aligned}
\boldsymbol{\alpha} & =\boldsymbol{x}_{i}-\boldsymbol{x}_{j}=\boldsymbol{\alpha}_{0}+\boldsymbol{\alpha}_{1} t \\
\boldsymbol{\beta} & =\boldsymbol{x}_{i+1}-\boldsymbol{x}_{i}=\boldsymbol{\beta}_{0}+\boldsymbol{\beta}_{1} t \\
\boldsymbol{\gamma} & =\boldsymbol{x}_{j+1}-\boldsymbol{x}_{j}=\gamma_{0}+\gamma_{1} t
\end{aligned}
$$

where

$$
\begin{aligned}
\boldsymbol{\alpha}_{0} & =\boldsymbol{x}_{i}^{n}-\boldsymbol{x}_{j}^{n} \\
\boldsymbol{\alpha}_{1} & =\boldsymbol{v}_{i}^{\Delta t}-\boldsymbol{v}_{j}^{\Delta t} \\
\boldsymbol{\beta}_{0} & =\boldsymbol{x}_{i+1}^{n}-\boldsymbol{x}_{i}^{n} \\
\boldsymbol{\beta}_{1} & =\boldsymbol{v}_{i+1}^{\Delta t}-\boldsymbol{v}_{i}^{\Delta t} \\
\gamma_{0} & =\boldsymbol{x}_{j+1}^{n}-\boldsymbol{x}_{j}^{n} \\
\gamma_{1} & =\boldsymbol{v}_{j+1}^{\Delta t}-\boldsymbol{v}_{j}^{\Delta t}
\end{aligned}
$$

The coefficients of the polynomial $V_{i j}=a_{3} t^{3}+a_{2} t^{2}+a_{1} t+a_{0}$ are then given by

$$
\begin{aligned}
& a_{0}=\boldsymbol{\alpha}_{\mathbf{0}} \cdot\left(\boldsymbol{\beta}_{\mathbf{0}} \times \gamma_{\mathbf{0}}\right) \\
& a_{1}=\boldsymbol{\alpha}_{\mathbf{0}} \cdot\left(\boldsymbol{\beta}_{\mathbf{1}} \times \gamma_{\mathbf{0}}\right)+\boldsymbol{\alpha}_{\mathbf{1}} \cdot\left(\boldsymbol{\beta}_{\mathbf{0}} \times \gamma_{\mathbf{0}}\right)+\boldsymbol{\alpha}_{\mathbf{0}} \cdot\left(\boldsymbol{\beta}_{\mathbf{0}} \times \gamma_{\mathbf{1}}\right) \\
& a_{2}=\boldsymbol{\alpha}_{\mathbf{1}} \cdot\left(\boldsymbol{\beta}_{\mathbf{1}} \times \gamma_{\mathbf{0}}\right)+\boldsymbol{\alpha}_{\mathbf{0}} \cdot\left(\boldsymbol{\beta}_{\mathbf{1}} \times \gamma_{\mathbf{1}}\right)+\boldsymbol{\alpha}_{\mathbf{1}} \cdot\left(\boldsymbol{\beta}_{\mathbf{0}} \times \gamma_{\mathbf{1}}\right) \\
& a_{3}=\boldsymbol{\alpha}_{\mathbf{1}} \cdot\left(\boldsymbol{\beta}_{\mathbf{1}} \times \gamma_{\mathbf{1}}\right)
\end{aligned}
$$

3c. Calculate $\boldsymbol{x}^{\tau}$, the bead positions at time $\tau$.

$$
\boldsymbol{x}^{\tau}=\boldsymbol{x}^{n}+\boldsymbol{v}^{\Delta t} \tau
$$


3d. Calculate $\boldsymbol{\chi}$, the point of intersection at time $\tau$, by solving the following set of equations:

$$
\chi=\boldsymbol{x}_{i}^{\tau}+\lambda_{i}\left(\boldsymbol{x}_{i+1}^{\tau}-\boldsymbol{x}_{i}^{\tau}\right)=\boldsymbol{x}_{j}^{\tau}+\lambda_{j}\left(\boldsymbol{x}_{j+1}^{\tau}-\boldsymbol{x}_{j}^{\tau}\right)
$$

where $\lambda_{i}$ and $\lambda_{j}$ define the point of intersection between the lines containing rods $i$ and $j$ respectively. If $\left(0 \leq \lambda_{i} \leq 1\right)$ and $\left(0 \leq \lambda_{j} \leq\right.$ 1) then the point of intersection lies on both rods and a rod-rod crossing has occurred. Otherwise, proceed to step 3k.

3e. Calculate $\boldsymbol{n}$, the unit vector normal to the plane formed by the two rods at time $\tau$

$$
\boldsymbol{n}=\frac{\left(\boldsymbol{x}_{i+1}^{\tau}-\boldsymbol{x}_{i}^{\tau}\right) \times\left(\boldsymbol{x}_{j+1}^{\tau}-\boldsymbol{x}_{j}^{\tau}\right)}{\left|\left(\boldsymbol{x}_{i+1}^{\tau}-\boldsymbol{x}_{i}^{\tau}\right) \times\left(\boldsymbol{x}_{j+1}^{\tau}-\boldsymbol{x}_{j}^{\tau}\right)\right|}
$$

3f. Calculate $\boldsymbol{v}_{\text {rel }}^{\Delta t}$, the relative velocity of the intersection point over the current time step

$$
\boldsymbol{v}_{\text {rel }}^{\Delta t}=\left(\boldsymbol{v}_{j}^{\Delta t}-\boldsymbol{v}_{i}^{\Delta t}\right)+\lambda_{j}\left(\boldsymbol{v}_{j+1}^{\Delta t}-\boldsymbol{v}_{j}^{\Delta t}\right)-\lambda_{i}\left(\boldsymbol{v}_{i+1}^{\Delta t}-\boldsymbol{v}_{i}^{\Delta t}\right)
$$

3g. Calculate $\boldsymbol{v}^{\text {col }}$, bead velocities after collision

$$
\begin{aligned}
\boldsymbol{v}_{i}^{\mathrm{col}} & =\boldsymbol{v}_{i}^{\Delta t}+2\left(1-\lambda_{i}\right)\left(\boldsymbol{n} \cdot \boldsymbol{v}_{\mathrm{rel}}^{\Delta t}\right) \boldsymbol{n} \\
\boldsymbol{v}_{i+1}^{\mathrm{col}} & =\boldsymbol{v}_{i+1}^{\Delta t}+2 \lambda_{i}\left(\boldsymbol{n} \cdot \boldsymbol{v}_{\mathrm{rel}}^{\Delta t}\right) \boldsymbol{n} \\
\boldsymbol{v}_{j}^{\mathrm{col}} & =\boldsymbol{v}_{j}^{\Delta t}-2\left(1-\lambda_{j}\right)\left(\boldsymbol{n} \cdot \boldsymbol{v}_{\mathrm{rel}}^{\Delta t}\right) \boldsymbol{n} \\
\boldsymbol{v}_{j+1}^{\mathrm{col}} & =\boldsymbol{v}_{j+1}^{\Delta t}-2 \lambda_{j}\left(\boldsymbol{n} \cdot \boldsymbol{v}_{\mathrm{rel}}^{\Delta t}\right) \boldsymbol{n}
\end{aligned}
$$

3h. Update positions for beads $i, i+1, j$, and $j+1$

$$
\boldsymbol{x}^{\ddagger}=\boldsymbol{x}^{\tau}+(\Delta t-\tau) \boldsymbol{v}^{\text {col }}
$$

3i. Calculate $\boldsymbol{v}_{\text {rel }}^{\dagger}$, the relative velocity of the intersection point using velocities at the end of time step

$$
\boldsymbol{v}_{\text {rel }}^{\dagger}=\left(\boldsymbol{v}_{j}^{\dagger}-\boldsymbol{v}_{i}^{\dagger}\right)+\lambda_{j}\left(\boldsymbol{v}_{j+1}^{\dagger}-\boldsymbol{v}_{j}^{\dagger}\right)-\lambda_{i}\left(\boldsymbol{v}_{i+1}^{\dagger}-\boldsymbol{v}_{i}^{\dagger}\right)
$$

3j. Update bead velocities at the end of the time step

$$
\begin{aligned}
\boldsymbol{v}_{i}^{\ddagger} & =\boldsymbol{v}_{i}^{\dagger}+2\left(1-\lambda_{i}\right)\left(\boldsymbol{n} \cdot \boldsymbol{v}_{\mathrm{rel}}^{\dagger}\right) \boldsymbol{n} \\
\boldsymbol{v}_{i+1}^{\ddagger} & =\boldsymbol{v}_{i+1}^{\dagger}+2 \lambda_{i}\left(\boldsymbol{n} \cdot \boldsymbol{v}_{\mathrm{rel}}^{\dagger}\right) \boldsymbol{n} \\
\boldsymbol{v}_{j}^{\ddagger} & =\boldsymbol{v}_{j}^{\dagger}-2\left(1-\lambda_{j}\right)\left(\boldsymbol{n} \cdot \boldsymbol{v}_{\mathrm{rel}}^{\dagger}\right) \boldsymbol{n} \\
\boldsymbol{v}_{j+1}^{\ddagger} & =\boldsymbol{v}_{j+1}^{\dagger}-2 \lambda_{j}\left(\boldsymbol{n} \cdot \boldsymbol{v}_{\mathrm{rel}}^{\dagger}\right) \boldsymbol{n}
\end{aligned}
$$


3k. If no collision occurs, then for beads $i, i+1, j$, and $j+1$

$$
\begin{aligned}
& \boldsymbol{v}^{\ddagger}=\boldsymbol{v}^{\dagger} \\
& \boldsymbol{x}^{\ddagger}=\boldsymbol{x}^{\dagger}
\end{aligned}
$$

4. Calculate the motion subject to the bead-surface uncrossability constraint to obtain $\boldsymbol{x}^{n+1}$ and $\boldsymbol{v}^{n+1}$.

This 3D algorithm can be extended to $2 \mathrm{D}$ by considering bead-rod collisions where the bead is treated as a rod perpendicular to the $2 \mathrm{D}$ plane with its position on the $2 \mathrm{D}$ plane.

\subsection{Algorithm Error}

For the bead-surface uncrossability constraint, equation (20) in [30] is incorrect. It updates the bead positions using the end-of-time-step velocities $\boldsymbol{v}^{n+1}$. The corrected equations should be

$$
\begin{gathered}
\boldsymbol{v}^{\Delta t}=\left(\boldsymbol{x}^{\dagger}-\boldsymbol{x}^{n}\right) / \Delta t \\
\boldsymbol{v}^{\mathrm{col}}=\boldsymbol{v}^{\Delta t}-2\left(\boldsymbol{n} \cdot \boldsymbol{v}^{\Delta t}\right) \boldsymbol{n} \\
\boldsymbol{x}^{n+1}=\boldsymbol{\chi}+(\Delta t-\tau) \boldsymbol{v}^{\mathrm{col}} .
\end{gathered}
$$

Note: These equations are actually the ones used in the original version of the code that I was given.

\subsection{Repulsive Potential}

The algorithm is taken from the work of Kumar and Larson [16]. The shortest vector between two rods is calculated, and a repulsive force is applied to both rods along that vector. While Kumar and Larson explored the use of an exponential potential and a Lennard-Jones potential, this work uses a short-ranged Debye-Hückel potential as defined in equation 4.

For algorithm details, please see [16]. It should be noted, however, that while the mathematically correct introduction of these forces is directly into the Langevin equation, I did not do it this way because I do not understand the math involved in the Ito-Taylor expansion. Therefore, I introduced these forces after the rod length constraint.

For polymer-surface interactions, the same algorithm was applied using the shortest vector between a rod and the level set boundary defining a surface. 


\section{Code}

Additions to the code were made in the files conTest.cpp and EBBroker.H. All additions begin with "////// BYBEE START" and end with "////// BYBEE END". All of these changes are accessible through the following parameters in the input file:

\section{1. constantFluid}

0: After particles are introduced, perform steps 1-4 (original version).

1: After particles are introduced, only perform step 1 (skip fluid solves).

2. particle.SAW

0: Particles are introduced in a straight line with the polymer position specified by particle.head_position and particle.tail_position (original version).

1: Particles are introduced as a self-avoiding walk with the polymer position specified by particle.center_of mass and the minimum distance between non-adjacent rods specified by particle.SAWmin_dist.

3. particle.self_interactions

0: Particles do not interact with each other (original version).

1: Particles do interact with each other through a rigid rod-rod uncrossability constraint.

2: Particles do interact with each other through a rod-rod DebyeHückel potential of the form $U=A \exp (-\kappa r) / r$. The parameters $A$ and $\kappa$ are specified by particle.self_int_A and particle.self_int k respectively. The potential is evaluated only for separations less than a cutoff specified by particle.self_int_cutoff.

4. particle.surface_interactions

0: Particles do not interact with surfaces.

1: Particles do interact with surfaces through a rigid bead-surface uncrossability constraint (original version).

2: Particles do interact with surfaces through a rod-surface DebyeHückel potential of the form $U=A \exp (-\kappa r) / r$. The parameters $A$ and $\kappa$ are specified by particle.surface_int_A and particle.surface_int_k 
respectively. The potential is evaluated only for separations less than a cutoff specified by particle.surface_int_cutoff.

\section{Results}

1. test_noFE

100-bead polymer. No polymer-polymer interactions, rigid constraint for polymer-surface interactions. Older simulation with old parameters.

2. test_FE

Same 100-bead polymer as test_noFE. Repulsive potential for polymerpolymer interactions, rigid constraint for polymer-surface interactions. Older simulation with old parameters.

3. test_02

200-bead polymer. Repulsive potential for both polymer-polymer and polymer-surface interactions. Due to the low grid resolution, the difference between the embedded boundary and the level set boundary is visually obvious. The polymer-surface interactions are based on the level set boundary.

4. test_03

200-bead polymer. Repulsive potential for both polymer-polymer and polymer-surface interactions. The grid resolution is higher than in test_02, and the difference between the embedded boundary and the level set boundary is not visually obvious.

5. test_04

Same 200-bead polymer as test_03. Rigid constraint for both polymerpolymer and polymer-surface interactions. As expected, the polymer gets closer to the pillar with the rigid constraint than with the repulsive potential. Due to lower fluid velocities near the pillar surface, the polymer moves more slowly.

This simulation exited with a floating point exception during the rod length constraint routine. 
6. test_05

Repeat of test_03 with the repulsive potential reduced by a factor of 100. As expected, the polymer gets closer to the pillar, and due to lower fluid velocities near the pillar surface, the polymer moves more slowly.

7. test_06

100-bead polymer. No polymer-polymer interactions, rigid constraint for polymer-surface interactions. (Not run to completion)

8. test_07

Same 100-bead polymer as test_06. Rigid constraint for both polymerpolymer and polymer-surface interactions. (Not run to completion)

9. test_08

Same 100-bead polymer as test_06. Repulsive potential for both polymerpolymer and polymer-surface interactions. (Not run)

\section{Discussion}

1. The following parameters were updated to reflect the correct use of the Kuhn step in the bead-rod model: $a=100 \mathrm{~nm}, m=3 \times 10^{-19}$, $m \gamma=1 \times 10^{-6}$.

2. In general, as the number of beads in the polymer increased, the parameter particle.cfl had to be decreased in order to maintain convergence in the constant rod length constraint routine. I think this is due to the beads being in a greater range of fluid velocities resulting in more stretching along the rods. For 100-bead polymers, a value of 1.0 seemed to work okay, and for 200-bead polymers, a value of 0.1 seemed to work okay.

3. Currently, the rod-rod uncrossability constraint loops through all rodrod pairs treating collisions as they are found. This does not always lead to correct results. The algorithm still needs to be modified so that it searches all rod-rod pairs for the first occuring collision, treats it, then searches all rod-rod pairs for the next occuring collision, treats it, etc., being careful to maintain the proper $\boldsymbol{v}^{\Delta t}$ and $\boldsymbol{v}^{\mathrm{col}}$. 
4. Combining the rod-rod and rod-surface uncrossability constraints into the same algorithm would be even more correct.

5. Are there exceptional cases not accounted for by the rod-rod uncrossability constraint? Parallel rods?

6. Does the rod-rod uncrossability constraint conserve kinetic energy?

7. The forces resulting from potential interactions should be introduced directly into the Langevin equation. I did not do it this way because I do not understand the math involved in the Ito-Taylor expansion. Therefore, I introduced these forces after the rod length constraint.

8. The Verlet neighbor list [1] has not been implemented for the rigid constraint or the smooth potential.

9. Adaptive time stepping. When the constant rod length constraint diverges or exceeds a maximum number of iterations, the time step could be divided into several shorter steps and repeated. How might this affect the statistics of the Brownian process?

10. My additions to the code should work for 3D without any modifications, although it has not been tested.

11. The smooth potential is much less computationally expensive than the rigid constraint. Potentials need only be evaluated once per rod pair, whereas the rigid constraint must loop over and over until all collisions have been treated.

\section{Previous Issues:}

1. I think neglecting particle inertia is appropriate. The phenomenological relaxation time for the particles is $1 / \gamma=3 \times 10^{-13}$ whereas the time step employed by the simulation is about $\Delta t=10^{-7}$.

A couple of benefits. First, the integration is more simple. Second, the constant rod length constraint was originally developed for systems with negligible particle inertia, and is mathematically correct for such systems. The mathematics get messed up once particle inertia is introduced. 
2. In the presence of hydrodynamic interactions, a polymer would not "bounce" off a pillar, but it would slow down due to the diverging resistance (lubrication theory).

3. The Brownian force is not exerted by the continuum fluid model but should be modeled as an external force. Therefore, only the drag force should be coupled back to the fluid.

\section{References}

[1] M. P. Allen and D. Tildesley. Computer Simulation of Liquids. Oxford, 1987.

[2] J. E. Butler and E. S. G. Shaqfeh. Brownian dynamics simulations of a flexible polymer chain which includes continuous resistance and multi-body hydrodynamic interactions. Journal of Chemical Physics, 122:014901, 2005.

[3] G. Ciccotti, M. Ferrario, and J. P. Ryckaert. Molecular dynamics of rigid systems in cartesian coordinates: a general formulation. Molecular Physics, 47:1253-1264, 1982.

[4] G. Ciccotti and J. P. Ryckaert. Molecular dynamics simulation of rigid molecules. Computer Physics Reports, 4:345, 1986.

[5] S. L. Dance and M. R. Maxey. Incorporation of lubrication effects into the force-coupling method for particulate two-phase flow. Journal of Computational Physics, 189(1):212-238, 2003.

[6] P. S. Doyle, E. S. G. Shaqfeh, and A. P. Gast. Dynamic simulation of freely-draining, flexible polymers in steady linear flows. Journal of Fluid Mechanics, 334:251-291, 1997.

[7] R. M. Jendrejack, J. J. de Pablo, and M. D. Graham. Macromolecules in microdevices: Multiscale simulation of DNA dynamics in model microfluidic geometries. In Nanotech, volume 1, chapter 2, 2002.

[8] R. M. Jendrejack, J. J. de Pablo, and M. D. Graham. Stochastic simulations of DNA in flow: Dynamics and the effects of hydrodynamic interactions. Journal of Chemical Physics, 116(17):7752-7759, 2002. 
[9] R. M. Jendrejack, E. T. Dimalanta, D. C. Schwartz, M. D. Graham, and J. J. de Pablo. DNA dynamics in a microchannel. Physical Review Letters, 91(3):038102, 2003.

[10] R. M. Jendrejack, D. C. Schwartz, J. J. de Pablo, and M. D. Graham. Shear-induced migration in flowing polymer solutions: Simulation of long-chain DNA in microchannels. Journal of Chemical Physics, 120(5):2513-2529, 2004.

[11] R. M. Jendrejack, D. C. Schwartz, M. D. Graham, and J. J. de Pablo. Effect of confinement on DNA dynamics in microfluidic devices. Journal of Chemical Physics, 119(2):1165-1173, 2003.

[12] H. Jian and A. V. Vologodski. A combined wormlike-chain and bead model for dynamic simulations of long linear DNA. Journal of Computational Physics, 136(168-179), 1997.

[13] F. Kienberger, V. P. Pastushenko, G. Kada, H. J. Gruber, C. Riener, H. Schindler, and P. Hinterdorfer. Static and dynamical properties of single poly(ethylene glycol) molecules investigated by force spectroscopy. Single Molecules, 2:123-128, 2000.

[14] K. Klenin, H. Merlitz, and J. Langowski. A Brownian dynamics program for the simulation of linear and circuler DNA and other wormlike chain polyelectrolytes. Biophysical Journal, 74:780-788, 1998.

[15] H. A. Kramers. The behavior of macromolecules in inhomogeneous flow. Journal of Chemical Physics, 14:415-424, 1946.

[16] S. Kumar and R. G. Larson. Brownian dynamics simulations of flexible polymers with spring-spring repulsions. Journal of Chemical Physics, 114(15):6937-6941, 2001.

[17] R. G. Larson. The Structure and Rheology of Complex Fluids. Oxford University Press, New York, 1999.

[18] D. Liu, M. Maxey, and G. E. Karniadakis. A fast method for particulate microflows. Journal of Microelectromechanical Systems, 11(6):691-702, 2002. 
[19] T. W. Liu. Flexible polymer chain dynamics and rheological properties in steady flows. Journal of Chemical Physics, 90:5826-5842, 1989.

[20] S. Lomholt, B. Stenum, and M. R. Maxey. Experimental verification of the force coupling method for particulate flows. International Journal of Multiphase Flow, 28:225-246, 2002.

[21] H. Meyer, O. Biermann, R. Faller, D. Reith, and F. Müller-Plathe. Coarse graining of nonbonded inter-particle potentials using automatic simplex optimization to fit structural properties. Journal of Chemical Physics, 113(6264), 2000.

[22] S. P. Mielke, W. H. Fink, V. V. Krishnan, N. Grønbech-Jensen, and C. J. Benham. Transcription-driven twin supercoiling of a DNA loop: A Brownian dynamics study. J. Chem. Phys., 121:8104-8112, 2004.

[23] J. T. Padding and W. J. Briels. Uncrossability constraints in mesoscopic polymer melt simulations: Non-Rouse behavior of $\mathrm{C}_{120} \mathrm{H}_{242}$. Journal of Chemical Physics, 115(6):2846-2859, 2001.

[24] P. D. Patel and E. S. G. Shaqfeh. A computational study of DNA separations in sparse disordered and periodic arrays of posts. Journal of Chemical Physics, 118(6):2941-2951, 2002.

[25] K. Rippe. Making contacts on a nucleic acid polymer. Trends in Biochemical Sciences, 26(12):733-740, 2001.

[26] W. B. Russel, D. A. Saville, and W. R. Schowalter. Colloidal Dispersions. Cambridge University Press, 1989.

[27] T. Schlick, D. A. Beard, J. Huang, D. A. Strahs, and X. L. Qian. Computational challenges in simulating large DNA over long times. Computing in Science and Engineering, 2(6):38-51, 2000.

[28] M. Somasi, B. Khomami, N. J. Woo, J. S. Hur, and E. S. G. Shaqfeh. Brownian dynamics simulations of bead-rod and bead-spring chains: numerical algorithms and coarse-graining issues. Journal of NonNewtonian Fluid Mechanics, 108:227-255, 2002.

[29] M. A. Streek. Brownian Dynamics Simulation of Migration of DNA in Structured Microschannels. PhD thesis, Universität Bielefeld, 2005. 
[30] D. Trebotich, G. H. Miller, P. Colella, D. T. Graves, D. F. Martin, and P. O. Schwartz. A tightly couple particle-fluid model for DNA-laden flows in complex microscale geometries. Computational Fluid and Solid Mechanics, pages 1018-1022, 2005.

[31] J.-L. Viovy. Electrophoresis of DNA and other polyelectrolytes: Physical mechanisms. Reviews of Modern Physics, 72(3):813-872, 2000.

[32] T. O. White, G. Ciccotti, and J. P. Hansen. Brownian dynamics with constraints. Molecular Physics, 99:2023-2036, 2001.

[33] N. J. Woo, E. S. G. Shaqfeh, and B. Khomami. The effect of confinement on dynamics and rheology of deoxyribose nucleic acid solutions. II. effective rheology and single chain dynamics. Journal of Rheology, 48(2):299-318, 2004.

[34] N. J. Woo, E. S. G. Shaqfeh, and B. Khomami. Effects of confinement on dynamics and rheology of dilute DNA solutions. I. entropic spring force under confinement and a numerical algorithm. Journal of Rheology, 48(2):281-298, 2004. 